\title{
Evolutionary Mechanisms of Cancer Suggest Rational Therapeutic Approaches
}

\author{
Alessio Balzerano Elena Paccosi Luca Proietti-De-Santis \\ Unit of Molecular Genetics of Aging, Department of Ecology and Biology, University of Tuscia, Viterbo, Italy
}

\section{Keywords}

Cancer (epi)genomic landscapes · Intratumor

heterogeneity - Cancer evolution - Liquid biopsy .

Circulating tumor DNA

\begin{abstract}
The goal in personalized therapeutic approaches for cancer medicine is to identify specific mutations with prognostic and therapeutic value in order to tailor the therapy for the single patient. The most powerful obstacle for personalized medicine arises from intratumor heterogeneity and clonal evolution, which can promote drug resistance. In this scenario, new technologies, such as next-generation sequencing, have emerged as a central diagnostic tool to profile cancer (epi)genomic landscapes. Therefore, a better understanding of the biological mechanisms underlying cancer evolution is mandatory and represents the current challenge to accurately predict whether cancer will recur after chemotherapy with the aim to tailor rational therapeutic approaches.

(c) 2021 S. Karger AG, Basel
\end{abstract}

\section{A.B. and E.P. are co-first authors.}

(C) 2021 S. Karger AG, Basel

www.karger.com $/ \mathrm{cg}$

\section{Introduction}

The hallmarks of cancer have been well defined from a biochemical and molecular genetics point of view [Hanahan and Weinberg, 2011]. They include sustaining proliferative signaling, evading growth suppressors, activating invasion and metastasis, enabling replicative immortality, inducing angiogenesis, and resisting cell death. It is well-known that tumor cells progressively acquire these new characteristics through a specific combination of somatic modifications at both the genetic and epigenetic level. However, these alterations are very different between each tumor, even of the same histological type. This heterogeneity is so extended that it is not thought that 2 tumors have an identical (epi)genetic profile [Stratton et al., 2009]. The recognition of the inter-tumor heterogeneity led to the concept of precision cancer medicine, which is aimed to identify specific mutations with prognostic (actionable) and therapeutic (druggable) value in order to tailor the therapy on the single patient. Along this line, next-generation sequencing technologies have emerged as a central diagnostic tool to profile these (epi)genomic landscapes. Despite the undisputed advances of personal therapy approaches in the care of some

Correspondence to:

Luca Proietti-De-Santis, proietti@unitus.it 
tumor types, the effectiveness of targeted therapies is often only transient because, almost invariably, tumors acquire drug resistance. The ability to predict whether and how long an individual tumor will respond to therapy and which genotype will evolve to drive resistance remains suboptimal [Dienstmann et al., 2013]. Therefore, the full understanding of the biological mechanisms behind cancer relapse is mandatory, as well as the identification of prognostic factors that may help to forecast the evolution of the cancer. The current challenge is to accurately predict the risk of cancer recurrence after potentially curative therapy and to support the oncologist in planning a different and more effective therapy.

\section{Mechanisms of (Epi)Genetic Variability in Cancer}

A key feature of cancer biology is its intratumor heterogeneity (ITH), characterized by genetic and epigenetic variability of somatic cells. The presence of genomic diversity within a single tumor was described for the first time in 1958 [Huxley, 1958]. However, only technological advances in the recent years, such as single-cell sequencing and subclonal tumor-region-specific genotyping, have enabled researchers to measure the entity of ITH. Johnson et al. [2014] have proposed that from 0 to over 8,000 different coding mutations exist within the same primary tumor or between primary and metastatic stages.

Such heterogeneity underlies the lethal outcome of cancer, therapeutic failure, and drug resistance. Moreover, ITH represents a fruitful background upon which the darwinian evolution proposed by Nowell in the 1970 s can act [Nowell, 1976]. The evolutionary mechanisms of cancer and the resulting heterogeneity are mainly characterized by 2 traits: (1) the presence of clones and subclones in a single tumor and (2) an ongoing genomic instability that works upon the landscape of pre-existing heterogeneity [McGranahan and Swanton, 2017].

The combination of both features introduces significant and increasing challenges in developing effective therapeutic and preventive strategies in fighting against cancer. In the now accepted model of cancer evolution, tumors undergo continual genetic and epigenetic mutational processes. Heritable alterations in sequence, structure, and regulation of DNA can be stochastically acquired by individual cells. Thus, as it occurs in the darwinian process for the evolution of the species, such alterations can increase the fitness of a cancer subclone, resulting in its expansion within the cancer cell population. This results in both temporally and spatially distinct

Cancer: Evolution and Therapy evolutionary trajectories among the different subclones [Burrell et al., 2013].

The parallel evolution of tumor subclones may lead to such a deep ITH that DNA copy number alteration observed in a single tumor biopsy may be characterized by profiles more similar to those of tumors from different patients than to those of adjacent biopsies [Martinez et al., 2013].

The somatic mutations underlying carcinogenesis are the consequence of physiological errors in the DNA replication machinery, defects in DNA repair pathways, lesions that are refractory to DNA repair, exposure to endogenous or exogenous mutagenic agents, and enzymatic modification of DNA. The genomic instability derived by these mechanisms represents the primary challenge in chemotherapy [McGranahan and Swanton, 2017]. On the other hand, cancer therapies can contribute themselves to genomic instability. For example, the sequencing of the exomes of 23 gliomas showed the role of the chemotherapeutic drug temozolomide (TMZ) in promoting a mutational pattern in the relapsing tumors, which is highly different from that of the primary tumors [Martinez et al., 2013].

Large-scale sequencing projects, such as The Cancer Genome Atlas (TCGA), the International Cancer Genome Consortium (ICGC), and the Cancer Genome Project (CGP), have enabled researchers to sequence tens of thousands of cancer genomes, thus generating a huge amount of data [Futreal et al., 2004; Feinberg et al., 2006; Hudson et al., 2010]. Importantly, from these data has emerged that not all mutations observed in cancer genomes contribute to the development and the evolution of tumoral phenotype. This led to distinguish between driver mutations and passenger mutations. Mutations that confer a proliferative advantage to cancer cells are defined driver. In contrast, passenger mutations are considered those mutations that do not necessarily determine the development of the cancer [Stratton et al., 2009; Pon and Marra, 2015].

Both genetic, ranging from small DNA sequence alterations to structural variations, and epigenetic mutations may affect driver genes and contribute to the development and progression of cancer.

Next-generation sequencing analyses highlighted that point mutation events often occur in specific and recurring combinations, termed "signatures." Currently, mutational signatures are categorized into 3 different classes of mutations: single-base substitutions, doublet-base substitutions, and small insertions and deletions. Each of these classes comprises several mutational signatures, 
characterized by high variability in both etiology and prevalence across human cancer types [Alexandrov et al., 2020]. A remarkable example is the one attributed to over-activity of members of the APOBEC family of cytidine deaminases [Burns et al., 2013; Taylor et al., 2013; Chan et al., 2015], characterized by the prominence of $\mathrm{C}>\mathrm{T}$ substitutions in $\mathrm{TpCpN}$ trinucleotides. This signature was observed in cancers arising from different tissues of origin, both solid (including bladder, breast, lung, as well as pancreas) and blood-derived (such as acute lymphoblastic leukemia and chronic lymphocytic leukemia) [Alexandrov et al., 2013]. In contrast, other signatures remain confined to one or a few cancer types, such as the one attributed to ultraviolet light in malignant melanoma [Pfeifer et al., 2005; Alexandrov et al., 2020].

In addition to these genome-wide mutational signatures, specific clusters of localized hypermutation termed kataegis, were recently observed in different cancer types, including breast and bone tumors [Nik-Zainal et al., 2012; D'Antonio et al., 2016; Franceschini et al., 2020]. The diversity of mutational processes underlying the development of cancer has potential implications for the understanding of cancer etiology, prevention, and therapy.

In carcinogenesis, somatic structural variations are defined as the set of structural rearrangements of DNA detectable in cancer cells, but not in healthy cells from the same patient [Tubio, 2015]. Structural variations usually manifest as breakage and subsequent fusion of genomic material, ranging from a single gene to whole chromosomes, and result in a plethora of alterations. Among them, copy number variations (i.e., amplifications and deletions), intra- and interchromosomal rearrangements, and insertion of transposable elements or exogenous DNA, such as viral and mitochondrial DNA [Li et al., 2020], are the most common. As described for base substitutions and indel mutations, also somatic structural alterations are characterized by marked variability between both different cancer types and within the same tumor [Yang et al., 2014]. Even if not all structural variants are directly involved in the processes of birth and development of cancer [Pon and Marra, 2015], some of them may act as driver mutations by altering the normal functioning or expression of key genes. Events that lead to the creation of fusion genes, duplication of oncogenes or translocation in a more transcriptional permissive genomic region represent the more common examples [Kurzrock et al., 2003; Crasta et al., 2012; Ott et al., 2013; Tubio, 2015]. The Philadelphia chromosome $(\mathrm{Ph})$ is among the most famous examples of chromosomal rearrangement resulting in gene fusions. The Philadelphia chromosome consists of a recip- rocal translocation of genetic material between chromosome 9 and chromosome 22. This translocation leads to the fusion of 2 genes: $A B L$, on chromosome 9, and $B C R$, on chromosome 22. The fusion oncogene $B C R-A B L$ encodes an enzyme with abnormal tyrosine kinase activity, resulting in an uncontrolled cell division. The Philadelphia chromosome was found in $95 \%$ of cases of chronic myeloid leukemia (CML), as well as in 25 and $5 \%$ of adult and childhood acute lymphoblastic leukemia, respectively [Kurzrock et al., 2003]. A key gene, whose deregulation following structural rearrangements is frequently observed in a wide variety of cancer types, is MYC. Gene duplication, as a consequence of genome doubling or tandem duplications, represents the most commonly found mechanism of MYC dysregulation. The resulting copy number alteration of $M Y C$ oncogene was observed in many different tumor types, including breast, ovarian, colorectal, and lung tumors [Beroukhim et al., 2010; Kalkat et al., 2017]. In addition, alterations of $M Y C$ expression in cancer can be achieved by translocation events juxtaposing the MYC gene with highly transcribed genomic regions. For instance, the translocation of MYC in Burkitt lymphoma (a subtype of non-Hodgkin B-cell lymphoma) can bring MYC expression under control of the immunoglobulin heavy chain enhancer, resulting in an undesired constitutive expression of this oncogene [Meyer and Penn, 2008; Ott et al., 2013].

Three main mechanisms are involved in the onset of structural variations: homologous recombination, nonreplicative non-homologous repair, and replicationbased mechanisms [Gu et al., 2008; Hastings et al., 2009]. Mutations in genes involved in these mechanisms may boost chromosomal instability and contribute to cancer development. For example, tumor suppressor genes breast cancer 1 (BRCA1) and breast cancer 2 (BRCA2) play a key role in homologous recombination-mediated repair of double-strand DNA breaks. Mutations in these 2 genes are commonly observed in hereditary breast and ovarian cancers. About $10 \%$ of ovarian cancers and $3 \%$ of breast cancers result from inherited mutations in the $B R C A 1$ and BRCA2 genes [Venkitaraman, 2002].

Furthermore, somatic or inherited mutations in genes involved in the mismatch repair machinery may result in error-prone replication. Besides the addiction of wrong nucleotides, one of the possible outcomes of a defective DNA replication is the recombination of short DNA repeats, known as microsatellites. Their resulting dynamic expansion and contraction across the genome has been observed in 15\% of colon cancers [Umar et al., 2004; Arana and Kunkel, 2010]. 
Another replication-associated mechanism that may contribute to chromosome rearrangements and, hence, to the carcinogenesis process is called fork stalling and template switching (FoSTeS), also named microhomology-mediated break-induced replication (MMBIR) [Yang et al., 2014]. MMBIR occurs when a replication fork collapses, resulting in a single-ended double-strand break. The replication machinery responds to this condition by resecting the $5^{\prime}$ end of the DNA. At this point, the $3^{\prime}$ ssDNA so created is annealed via microhomology (2-5 nucleotides) to another nearby available ssDNA, such as the lagging strand of an upstream replication fork. MMBIRmediated rearrangements can take place during mitosis and can generate complex genomic alterations, such as duplications or triplications of individual genes and rearrangements of single exons [Zhang et al., 2009]. Another harmful consequence of MMBIR is the creation of double-minute chromosomes (DMs). DMs are small and circular fragments of extrachromosomal DNA, lacking centromeres and telomeres, that can duplicate autonomously. DMs have been revealed in a large number of human cancers, both solid, including lung and glioblastoma [Bigner et al., 1990; Nielsen et al., 1993], and blood-derived, such as CML and acute myeloid leukemia (AML) [Morel et al., 2003; Thomas et al., 2004]. DMs are often associated with oncogene amplification, that results in subclone selective advantage and drug resistance. For instance, in glioblastoma patients, DMs have been observed in $50 \%$ of cases, and the epidermal growth factor receptor gene (EGFR) represented the most commonly amplified gene [Bigner et al., 1990]. In AML and myelodysplastic syndrome, these small circular chromosomes have been found in about $1 \%$ of cases. Most of them showed an amplified segment from chromosome 8q24 harboring the $M Y C$ oncogene. Moreover, in CML patients, the amplification of oncogene fusion $B C R-A B L$ was associated with the presence of DMs containing $B C R-A B L$ fusion genes [Morel et al., 2003].

The role of a gradual acquisition of genetic mutations that activate oncogenes and/or inactivate tumor suppressor genes, as primary fuel of the cancerogenic process, is now well established [Stratton et al., 2009]. However, a percentage of cancers is characterized by a process that challenges this paradigm, termed chromothripsis. During chromothripsis, tens to hundreds of genomic rearrangements, clustered into discrete subchromosomal territories, occur in one single punctual event and result in a multitude of gene fusions with novel oncogenic potential and/or disruption of tumor suppressor genes [Stephens et al., 2011]. Two possible mechanisms are sug- gested for chromothripsis: chromosome end-to-end fusions following telomere shortening and DNA damage associated with the replication of chromosomes packaged into micronuclei [Crasta et al., 2012; Garsed et al., 2014]. Although the most expected outcome of these "genomic catastrophes" is cell death, an increasing number of studies showed that chromothripsis underlies an extensive remodeling of the genome promoting cancer development [Jones and Jallepalli, 2012; Li et al., 2014].

It is estimated that chromothripsis occurs in about $2-3 \%$ of all tumors, but it is characterized by high variability across cancer types: $30 \%$ of osteosarcoma samples, $18 \%$ of chordomas, $11 \%$ of neuroblastomas, $10 \%$ of chronic lymphocytic leukemias, and $1.3 \%$ of multiple myeloma [Tubio, 2015]. Interestingly, chromothripsis manifestations have also been observed in tumors of patients affected by Li-Fraumeni syndrome, a hereditary cancer predisposition first reported in 1969 by Frederick Li and Joseph Fraumeni, characterized by germline mutations in the TP53 tumor suppressor gene. The link between chromothripsis and TP53 deficiency is particularly evident in medulloblastomas, especially in a specific type of this cancer, named Sonic Hedgehog pathway ( $\mathrm{SHH}$ ) medulloblastoma. Rausch et al. [2012] reported that in SHH medulloblastoma harboring TP53 mutations, chromothripsis frequency varies from 30 to $100 \%$. By contrast, patients with wild-type TP53 alleles showed no events of chromothripsis. Likewise, $50 \%$ of AML patients with somatic TP53 mutations exhibited chromosomal rearrangements, whereas the percentage of wild-type TP53 samples with chromothripsis patterns is just $1 \%$. Noteworthy, the loss of p53 functionality can be determined not only by inactivating mutations of the same gene, but also by hyperactivation of proteins involved in its negative modulation [Proietti-DeSantis et al., 2003, 2018; Caputo et al., 2013, 2017].

In the traditional view, tumor heterogeneity is usually associated with genetic mutations and structural alterations. However, an increasing number of studies have highlighted the fundamental role that epigenomic alterations play within the complex evolution of cancer. Indeed, epigenetic variations may contribute to a marked intratumor variability, even when the tumor shows a high degree of genetic homogeneity, resulting in different evolutionary paths and responses to therapies [Kreso et al., 2013; Mazor et al., 2016]. Analysis of epigenetic alterations in prostate cancers, gliomas, and esophageal squamous cell carcinomas have shown deep ITH, whose magnitude is comparable to that observed at the genomic level [Aryee et al., 2013; Mazor et al., 2015; Hao et al., 2016]. Different epigenetic mechanisms can be associated with 
tumor heterogeneity, including changes in DNA methylation [Lister et al., 2009], altered histone modification patterns [Hake et al., 2004; Kurdistani, 2007], and chromatin remodeling [Rodríguez-Paredes and Esteller, 2011]. Alterations in methylation patterns, such as genome-wide DNA hypomethylation or site-specific CpG island promoter hypermethylation, are usually described in early stages of the carcinogenesis process. This highlights the primary role that the disruption of the epigenetic machineries may play in promoting cancer development and progression. Indeed, within the traditional 2-hit model, also known as Knudson hypothesis [Knudson, 1971], hypermethylation of a specific promoter may represent the second hit necessary to silence the remaining allele of a previously mutated tumor suppressor gene [Jones and Laird, 1999]. On the other hand, a global DNA hypomethylation has been proposed to be the cause underlying the chromosomal instability observed in a T-cell lymphoma in mice [Gaudet et al., 2003]. Irizarry et al. [2009] have observed that the majority of methylation alterations in colon cancer occur neither in $\mathrm{CpG}$ islands nor in gene promoters, but in sequences in a short distance from the CpG islands themselves, that they termed "CpG island shores." They showed that these methylation changes resemble those normally associated with tissue differentiation. This evidence is consistent with the epigenetic progenitor model, which asserts that epigenetic alterations, that affect the tissue-specific stem/progenitor cells and, subsequently, disrupt their normal differentiation, are at the basis of cancer development [Feinberg et al., 2006; Irizarry et al., 2009].

Specific alterations in DNA methylation can be associated with different patient outcomes. A remarkable example is the one involving DNA hypermethylation at the promoter of O6-methylguanine DNA methyltransferase (MGMT). MGMT is an enzyme involved in DNA repair that counteracts the effect of alkylating agents, such as the chemotherapeutic drug TMZ, by removing alkyl groups from guanine residues.

It has been observed that glioblastoma patients with normal MGMT gene expression do not usually respond to TMZ or other alkylating antineoplastic drug treatments. In contrast, epigenetic silencing of the MGMT gene by promoter hypermethylation in glioblastoma patients was associated with a favorable prognosis following chemotherapy [Weller et al., 2010]. For this reason, $M G M T$ promoter methylation is considered a strong prognostic biomarker for the sensitivity of glioblastoma patients to chemotherapy with alkylating agents [Hegi et al., 2005; Wick et al., 2014].

\section{The Fight against Cancer Variability: Cancer Evolution and Drug Resistance}

Cancer variability does not only occur in different patients with the same type of tumor (interpatient heterogeneity) but also in the same patient either within adjacent biopsies or among the multiple secondary metastases (intrapatient heterogeneity). Subpopulations of cancer cells with distinct phenotypic and molecular features within a tumor are called intratumor heterogeneity [Bedard et al., 2013].

This cancer variability is based on both the deterministic mechanism of selection and on stochastic processes, in which we can have different outcomes, even if the initial state is the same; examples of this kind of mechanisms are genetic drift and the acquisition of heritable alterations.

Nowell [1976] was the first to propose that the darwinian theory could be applied to cancer development and progression affirming that, in a neoplasm arising from a single cell of origin, tumor progression results from acquired genetic variability within the original clone allowing the sequential selection of more aggressive sublines; by then, it is universally known that, if tumor heterogeneity leads to a gain in terms of fitness and survival, it will be subject to a positive selection.

Cancer can be viewed as an ecological entity that dynamically interacts with the microenvironment and forms specialized niches to support its expansion. Cancer evolution is strictly related to niche composition and adaptation. The niche is a special local site in the stromal microenvironment of stem cells that integrates signals [Schofield and Weightman, 1978], regulates stem cell fate commitment and epithelial cell plasticity during tissue homeostasis and regeneration [Blanpain and Fuchs, 2014]. Tumor cells drastically shape and subvert the niche [Barcellos-Hoff et al., 2013], that is important for tumor cell hierarchy and regulation of cancer stem cell activities [Plaks et al., 2015].

In some cases, cancer evolution allows drug resistant diseases [Gerlinger and Swanton, 2010; Yates and Campbell, 2012] by improving tumor adaptation to therapy. Drug resistance causes less sensitivity to chemotherapy and is the result of the combined actions of multiple factors and pathways [Marin et al., 2016]. Drug resistance is determined by increased drug efflux, increased DNA damage repair, reduced apoptosis, modification or alteration of drug active target proteins, transformation of epithelial cells to mesenchymal cells and so on [Huang et al., 2016]. 
Also hypoxia can trigger drug resistance, especially in solid tumors, by affecting gene stability of tumor cells, upregulating glycolytic enzyme expression, downregulating adhesion molecules, and maintaining stem cell characteristics, so improving growth, invasiveness, and metastatisation abilities of tumor tissues [Kewitz et al., 2016; Lu and Kang, 2019].

Furthermore, the initial depletion in the cancer cell population, as happens after cytotoxic treatment, may imply a reduction of heterogeneity, leading to a phenomenon called "bottleneck effect:" despite initial therapeutic responsiveness, treatment can induce a bottleneck, in which the death of drug-sensitive cells is counteracted by a massive outgrowth of resistant cells that will survive, being resistant to the initial drug therapy used. So, the initial heterogeneic tumor population will be lost, and the fewdrug resistant cells will repopulate the tumor [Marusyk and Polyak, 2010].

This phenomenon will lead to a pronounced genetic drift, which is the change in the frequency of an allele in a population [Lynch, 2007; Levy et al., 2015]. While darwinian selection is a deterministic mechanism, the genetic drift is a stochastic process, instead. However, both of them contribute to cancer evolution and variability.

This is the case of AML, where some of the subclones observed at initial diagnosis are no longer detectable after chemotherapy [Ding et al., 2012], while newly emerging subclones, undetectable before therapy, appear after chemotherapy, indicating that heterogeneity may increase again during population expansion. Driver mutation landscapes often significantly differ from the initially detected driver mutations since clones had branched off early in tumor evolutionary history [Johnson et al., 2014].

In CML patients treated with imatinib, a tyrosine kinase inhibitor targeting the BCR-ABL1 oncoprotein [Goldman and Melo, 2001], a disease progression and resistance-caused treatment failure have been observed [Holyoake and Vetrie, 2017]. The secondary resistance is due to the evolution of leukemia cells under the pressure of drug treatment [Hehlmann et al., 2017], that leads to the formation of drug-resistant dominant clones [Pietarinen et al., 2017]. Progeny cells from these clones have a strong ability to proliferate and lose the ability to differentiate into relatively mature blood cells. Recent studies showed that the protective upregulation of $\mathrm{Wnt} / \beta$-catenin and $\mathrm{ABC}$ transporter signals induced by imatinib treatment is neutralized by knockdown of transcription factor 7 (TCF7), one of the main Wnt/ $\beta$-catenin signaling mediators [Zhang et al., 2020]. While uncontrolled cell proliferation that precedes cancer involves several cell clones, the crucial event leading to cancer is the selection and further expansion of a single clone, characterized by a "carcinogenic advantage" [Wright, 2002]. In fact, a new mutation, if able to improve cell surviving under particular environmental conditions and to escape drift, will became more and more abundant within the population [Jain and Krug, 2007; Szendro et al., 2013]. Furthermore, since multiple subclones within a tumor can lead to clonal competition, then the fitness of a single subclone will be defined in relation to the fitness of other competing clones, thus complicating the prediction of the evolutionary outcomes [Gerrish and Lenski, 1998].

If subclones evolve independently, they will differ from each other in terms of sensitivity to cancer drugs and impact on patient survival; it is important to accurately detect such subclones in order to improve clinical diagnosis and personalized medicine approaches [Haffner et al., 2013].

Regarding cancer evolutionary progression, tumorigenesis does not proceed only in a linear way, in which a clone showing advantageous driver mutations replaces the other clones, but also in a branched evolutionary way. The result of this branched tumor evolution and development is a number of subpopulations with different treatment response phenotypes than the original tumor-initiating cell. Tracing multiple subpopulations of cells to their most recent common ancestor using a phylogenetic tree is one way of modeling tumor evolution: in this model the trunk of the tree represents clonal driver events that occur early in tumor development, whereas the branches represent subclonal driver events that differ from one subpopulation to the other. A single ancestral clone can give rise to multiple subclones both in presence and absence of treatment pressure [Jamal-Hanjani et al., 2017].

This model provides an adaptation to different microenviroments and can explain the tumoral diversification and evolution in different kinds of human cancers, as acute leukemia [Notta et al., 2011], prostate cancer [Haffner et al., 2013], glioma [Johnson et al., 2014], medulloblastoma [Wu et al., 2012], and many kinds of carcinomas [Thirlwell et al., 2010; Nik-Zainal et al., 2012; Xu et al., 2012].

Multiple myeloma, for example, is characterized by branching patterns of subclonal evolution from reservoir clones with a propagating potential and/or the emergence of minor clones, which compete other clones through selective pressure determined mainly by therapeutic agents. Each subclone harbors novel mutations and distinct phenotypes, including drug sensitivities, so the branching evolution is a result of the fitness of different clones to 
microenvironment and their evasion of immune surveillance [Furukawa and Kikuchi, 2020].

A branched evolution pattern was also found in brain metastasis by exome sequencing. Even sharing a common ancestor, both the primary tumor and the metastasis continued to evolve separately [Brastianos et al., 2015].

Branched evolution is not a prerequisite for tumor development since some tumor types can develop in both linear and branched manners [Anderson et al., 2011].

Out of doubt, branched evolution and the overall evolutionary perspective of cancer have a big relevance in oncology, and their knowledge is mandatory for the understanding of the clonal dynamics during tumoral progression, in order to delineate a better approach for cancer medicine efforts.

\section{Monitoring Tumor Evolution Using Liquid Biopsy}

When solid tumors recur, 2 aspects are particularly challenging for the oncologist: (1) to identify the relapse as soon as possible in order to avoid multiple metastasis dissemination; and (2) to characterize the full genomic landscape of the relapsed cancer since its molecular profile is extremely complex. This complexity is due to the multiple and concomitant presence of low-frequency subclones. In fact, as described above, different subclones can determine the achievement of drug resistance through the evolution of either different evolutionary branches or a single dominant clone [Meacham and Morrison, 2013]. In particular, this phenomenon occurs when evolution is shaped by resistance to kinase inhibitors [Diaz et al., 2012; Murtaza et al., 2015]. Along this line, it appears clear that standard prognostic procedures, such as tissue biopsies, are inadequate to give a full comprehensive view of the genomic landscape, given that even subsets of the same metastases often reveal different genomic profiles. Likewise, tissue biopsy does not appear to be a feasible methodology in guaranteeing the repeated longitudinal profiling of the molecular landscapes that is required during treatment, because of the evolutive nature of cancer.

Recently, to respond to this need the "liquid biopsy" methodology has been developed, allowing the genotyping of DNA that circulates in the blood of patients. This may be achieved by new kinds of approaches such as nextgeneration sequencing and droplet digital PCR; both of them guarantee a sensitive and specific detection of low frequency [Alix-Panabières and Pantel, 2016]. Indeed, the blood of cancer patients contains both circulating tumor cells [Yu et al., 2011] and circulating tumor DNA
(ctDNA) [Jen et al., 2000] derived from primary tumors, metastatic lesions, or circulating tumor cells. Several studies have shown that mutations in ctDNA exactly recapitulate mutations identified in the tumor, including both genetic mutations and genomic alterations, such as copy-number changes and genome rearrangements [Leary et al., 2012; Murtaza et al., 2013; Bettegowda et al., 2014]. ctDNA levels are also known to correlate with disease progression, as a reduction in ctDNA levels is associated with successful pharmacological and/or surgical treatment [Diehl et al., 2008].

Currently, many studies convincingly demonstrate that the liquid biopsy will be in the near future the mandatory tool to both comprehensively assess the therapeutic responses in primary cancer and monitor disease recurrence in the post-treatment setting [Beaver et al., 2014; Tie et al., 2015; Alix-Panabières and Pantel, 2016; Chabon et al., 2016].

Along this line, diagnostic and therapeutic prediction models could be useful for personalized medicine. The support vector machine is a state-of-the-art machine learning approach that has been employed to forecast breast cancer subtypes using input data from transcriptomics, epigenetics, proteomics, radiomics, clinical, pathological, and biochemical analysis. Shortening the timeline required for both diagnosis and treatment of most aggressive cancers, this kind of machine learning techniques will have important clinical and public applications and implications for high-throughput data analysis and contextualization [Ozer et al., 2020].

\section{Conflict of Interest Statement}

The authors have no conflicts of interest to declare.

\section{Funding Sources}

The authors received no specific funding for this work.

\section{Author Contributions}

All authors contributed equally to this work. 


\section{References}

Alexandrov LB, Nik-Zainal S, Wedge DC, Aparicio SAJR, Behjati S, Biankin AV, et al. Signatures of mutational processes in human cancer. Nature. 2013;500:415-21.

Alexandrov LB, Kim J, Haradhvala NJ, Huang MN, Tian Ng AW, Wu Y, et al. The repertoire of mutational signatures in human cancer. Nature. 2020;578:94-101.

Alix-Panabières C, Pantel K. Clinical applications of circulating tumor cells and circulating tumor DNA as liquid biopsy. Cancer Discov. 2016;6(5):479-91.

Anderson K, Lutz C, van Delft FW, Bateman CM, Guo Y, Colman SM, et al. Genetic variegation of clonal architecture and propagating cells in leukaemia. Nature. 2011;469(7330):356-61.

Arana ME, Kunkel TA. Mutator phenotypes due to DNA replication infidelity. Semin Cancer Biol. 2010;20:304-11.

Aryee MJ, Liu W, Engelmann JC, Nuhn P, Gurel M, Haffner MC, et al. DNA methylation alterations exhibit intraindividual stability and interindividual heterogeneity in prostate cancer metastases. Sci Transl Med. 2013;5: $169 \mathrm{ra} 10$.

Barcellos-Hoff MH, Lyden D, Wang TC. The evolution of the cancer niche during multistage carcinogenesis. Nat Rev Cancer. 2013;13(7): 511-8.

Beaver JA, Jelovac D, Balukrishna S, Cochran RL, Croessmann S, Zabransky S, et al. Detection of cancer DNA in plasma of patients with early-stage breast cancer. Clin Cancer Res. 2014; 20:2643-50.

Bedard PL, Hansen AR, Ratain MJ, Siu LL. Tumour heterogeneity in the clinic. Nature. 2013;501(7467):355-64.

Beroukhim R, Mermel CH, Porter D, Wei G, Raychaudhuri S, Donovan J, et al. The landscape of somatic copy-number alteration across human cancers. Nature. 2010;463:899905.

Bettegowda C, Sausen M, Leary RJ, Kinde I, Wang Y, Agrawal N, et al. Detection of circulating tumor DNA in early- and late-stage human malignancies. Sci Transl Med. 2014;6: $224 \mathrm{ra} 24$.

Bigner SH, Mark J, Bigner DD. Cytogenetics of human brain tumors. Cancer Genet Cytogenet. 1990;47:141-54.

Blanpain C, Fuchs E. Stem cell plasticity. Plasticity of epithelial stem cells in tissue regeneration. Science. 2014;344(6189):1242281.

Brastianos PK, Carter SL, Santagata S, Cahill DP, Taylor-Weiner A, Jones RT, et al. Genomic Characterization of Brain Metastases Reveals Branched Evolution and Potential Therapeutic Targets. Cancer Discov. 2015;5(11):116477.

Burns MB, Lackey L, Carpenter MA, Rathore A, Land AM, Leonard B, et al. APOBEC3B is an enzymatic source of mutation in breast cancer. Nature. 2013;494:366-70.
Burrell RA, McGranahan N, Bartek J, Swanton C. The causes and consequences of genetic heterogeneity in cancer evolution. Nature. 2013; 501:338-45.

Caputo M, Frontini M, Velez-Cruz R, Nicolai S, Prantera G, Proietti-De-Santis L. The CSB repair factor is overexpressed in cancer cells, increases apoptotic resistance, and promotes tumor growth. DNA Repair (Amst). 2013; 12(4):293-9.

Caputo M, Balzerano A, Arisi I, D’Onofrio M, Brandi R, Bongiorni S, et al. CSB ablation induced apoptosis is mediated by increased endoplasmic reticulum stress response. PLoS One. 2017;12(3):e0172399.

Chabon JJ, Simmons AD, Lovejoy AF, Esfahani MS, Newman AM, Haringsma HJ, et al. Circulating tumour DNA profiling reveals heterogeneity of EGFR inhibitor resistance mechanisms in lung cancer patients. Nat Commun. 2016;7:11815.

Chan K, Roberts SA, Klimczak LJ, Sterling JF, Saini N, Malc EP, et al. An APOBEC3A hypermutation signature is distinguishable from the signature of background mutagenesis by APOBEC3B in human cancers. Nat Genet. 2015;47:1067-72.

Crasta K, Ganem NJ, Dagher R, Lantermann AB, Ivanova EV, Pan Y, et al. DNA breaks and chromosome pulverization from errors in $\mathrm{mi}$ tosis. Nature. 2012;482:53-8.

D’Antonio M, Tamayo P, Mesirov JP, Frazer KA. Kataegis Expression Signature in Breast Cancer Is Associated with Late Onset, Better Prognosis, and Higher HER2 Levels. Cell Rep. 2016;16:672-83.

Diaz LA, Williams RT, Wu J, Kinde I, Hecht JR, Berlin J, et al. The molecular evolution of acquired resistance to targeted EGFR blockade in colorectal cancers. Nature. 2012;486:53740.

Diehl F, Schmidt K, Choti MA, Romans K, Goodman S, Li M, et al. Circulating mutant DNA to assess tumor dynamics. Nat Med. 2008;14: 985-90.

Dienstmann R, Rodon J, Barretina J, Tabernero J. Genomic medicine frontier in human solid tumors: prospects and challenges. J Clin Oncol. 2013;31:1874-84.

Ding L, Ley TJ, Larson DE, Miller CA, Koboldt DC, Welch JS, et al. Clonal evolution in relapsed acute myeloid leukaemia revealed by whole-genome sequencing. Nature. 2012; 481(7382):506-10.

Feinberg AP, Ohlsson R, Henikoff S. The epigenetic progenitor origin of human cancer. Nat Rev Genet. 2006;7:21-33.

Franceschini N, Lam SW, Cleton-Jansen AM, Bovée JVMG. What's new in bone forming tumours of the skeleton? Virchows Arch. 2020; 476:147-57

Furukawa Y, Kikuchi J. Molecular basis of clonal evolution in multiple myeloma. Int J Hematol. 2020;111(4):496-511.
Futreal PA, Coin L, Marshall M, Down T, Hubbard T, Wooster R, et al. A census of human cancer genes. Nat Rev Cancer. 2004;4:177-83.

Garsed DW, Marshall OJ, Corbin VDA, Hsu A, DiStefano L, Schröder J, et al. The Architecture and Evolution of Cancer Neochromosomes. Cancer Cell. 2014;26:653-67.

Gaudet F, Hodgson JG, Eden A, Jackson-Grusby L, Dausman J, Gray JW, et al. Induction of tumors in mice by genomic hypomethylation. Science. 2003;300:489-92.

Gerlinger M, Swanton C. How Darwinian models inform therapeutic failure initiated by clonal heterogeneity in cancer medicine. Br J Cancer. 2010;103(8):1139-43.

Gerrish PJ, Lenski RE. The fate of competing beneficial mutations in an asexual population. Genetica. 1998;102-103(1-6):127-44.

Goldman JM, Melo JV. Targeting the BCR-ABL tyrosine kinase in chronic myeloid leukemia. N Engl J Med. 2001;344(14):1084-6.

Gu W, Zhang F, Lupski JR. Mechanisms for human genomic rearrangements. Pathogenetics. 2008; $1: 4$.

Haffner MC, Mosbruger T, Esopi DM, Fedor H, Heaphy CM, Walker DA, et al. Tracking the clonal origin of lethal prostate cancer. J Clin Invest. 2013;123(11):4918-22.

Hake SB, Xiao A, Allis CD. Linking the epigenetic 'language' of covalent histone modifications to cancer. Br J Cancer. 2004;90:761-9.

Hanahan D, Weinberg RA. Hallmarks of cancer: The next generation. Cell. 2011;144:646-74.

Hao JJ, Lin DC, Dinh HQ, Mayakonda A, Jiang YY, Chang C, et al. Spatial intratumoral heterogeneity and temporal clonal evolution in esophageal squamous cell carcinoma. Nat Genet. 2016;48:1500-7.

Hastings PJ, Lupski JR, Rosenberg SM, Ira G. Mechanisms of change in gene copy number. Nat Rev Genet. 2009;10:551-64.

Hegi ME, Diserens AC, Gorlia T, Hamou MF, de Tribolet N, Weller M, et al. MGMT Gene Silencing and Benefit from Temozolomide in Glioblastoma. N Engl J Med. 2005;352(10):997-1003.

Hehlmann R, Lauseker M, Saußele S, Pfirrmann M, Krause S, Kolb HJ, et al. Assessment of imatinib as first-line treatment of chronic myeloid leukemia: 10-year survival results of the randomized CML study IV and impact of non-CML determinants. Leukemia. 2017; 31(11):2398-406

Holyoake TL, Vetrie D. The chronic myeloid leukemia stem cell: stemming the tide of persistence. Blood. 2017;129(12):1595-606.

Huang M, Liu T, Ma P, Mitteer RA, Zhang Z, Kim HJ, et al. c-Met-mediated endothelial plasticity drives aberrant vascularization and chemoresistance in glioblastoma. J Clin Invest. 2016;126(5):1801-14.

Hudson TJ, Anderson W, Aretz A, Barker AD, Bell C, Bernabé RR, et al. International network of cancer genome projects. Nature. 2010;464:993-8. 
Huxley J. Biological Aspects of Cancer. NewYork: Harcourt, Brace and Company; 1958.

Irizarry RA, Ladd-Acosta C, Wen B, Wu Z, Montano $\mathrm{C}$, Onyango $\mathrm{P}$, et al. The human colon cancer methylome shows similar hypo- and hypermethylation at conserved tissue-specific CpG island shores. Nat Genet. 2009;41:17886.

Jain K, Krug J. Deterministic and stochastic regimes of asexual evolution on rugged fitness landscapes. Genetics. 2007;175(3):1275-88.

Jamal-Hanjani M, Wilson GA, McGranahan N, Birkbak NJ, Watkins TBK, Veeriah S, et al. Tracking the Evolution of Non-Small-Cell Lung Cancer. N Engl J Med. 2017;376(22): 2109-21.

Jen J, Wu L, Sidransky D. An overview on the isolation and analysis of circulating tumor DNA in plasma and serum. Ann N Y Acad Sci. 2000 906:8-12.

Johnson BE, Mazor T, Hong C, Barnes M, Aihara $\mathrm{K}, \mathrm{McLean} \mathrm{CY}$, et al. Mutational analysis reveals the origin and therapy-driven evolution of recurrent glioma. Science. 2014;343:18993.

Jones MJK, Jallepalli PV. Chromothripsis: Chromosomes in Crisis. Dev Cell. 2012;23:908-17.

Jones PA, Laird PW. Cancer epigenetics comes of age. Nat Genet. 1999;21:163-7.

Kalkat M, De Melo J, Hickman KA, Lourenco C, Redel C, Resetca D, et al. MYC deregulation in primary human cancers. Genes (Basel). 2017;8:2-30.

Kewitz S, Kurch L, Volkmer I, Staege MS. Stimulation of the hypoxia pathway modulates chemotherapy resistance in Hodgkin's lymphoma cells. Tumour Biol. 2016;37(6):8229-37.

Knudson AG. Mutation and cancer: statistical study of retinoblastoma. Proc Natl Acad Sci USA. 1971;68:820-3.

Kreso A, O'Brien CA, Van Galen P, Gan OI, Notta F, Brown AMK, et al. Variable clonal repopulation dynamics influence chemotherapy response in colorectal cancer. Science. 2013;339:543-8.

Kurdistani SK. Histone modifications as markers of cancer prognosis: A cellular view. Br J Cancer. 2007;97:1-5.

Kurzrock R, Kantarjian HM, Druker BJ, Talpaz M. Philadelphia Chromosome-Positive Leukemias: From Basic Mechanisms to Molecular Therapeutics. Ann Intern Med. 2003;138: 819-30.

Leary RJ, Sausen M, Kinde I, Papadopoulos N, Carpten JD, Craig D, et al. Detection of chromosomal alterations in the circulation of cancer patients with whole-genome sequencing. Sci Transl Med. 2012;4:162ra154.

Levy SF, Blundell JR, Venkataram S, Petrov DA, Fisher DS, Sherlock G. Quantitative evolutionary dynamics using high-resolution lineage tracking. Nature. 2015;519(7542):181-6.

Li Y, Schwab C, Ryan SL, Papaemmanuil E, Robinson HM, Jacobs P, et al. Constitutional and somatic rearrangement of chromosome 21 in acute lymphoblastic leukaemia. Nature. 2014 508:98-102.
Li Y, Roberts ND, Wala JA, Shapira O, Schumacher SE, Kumar K, et al. Patterns of somatic structural variation in human cancer genomes. Nature. 2020;578:112-21.

Lister R, Pelizzola M, Dowen RH, Hawkins RD, Hon G, Tonti-Filippini J, et al. Human DNA methylomes at base resolution show widespread epigenomic differences. Nature. 2009; 462:315-22.

Lu W, Kang Y. Epithelial-Mesenchymal Plasticity in Cancer Progression and Metastasis. Dev Cell. 2019;49(3):361-74.

Lynch $M$. The evolution of genetic networks by non-adaptive processes. Nat Rev Genet. 2007; 8(10):803-13

Marin JJG, Al-Abdulla R, Lozano E, Briz O, Bujanda L, Banales JM, et al. Mechanisms of Resistance to Chemotherapy in Gastric Cancer. Anticancer Agents Med Chem. 2016;16(3): 318-34.

Martinez P, Birkbak NJ, Gerlinger M, McGranahan N, Burrell RA, Rowan AJ, et al. Parallel evolution of tumour subclones mimics diversity between tumours. J Pathol. 2013;230: 356-64.

Marusyk A, Polyak K. Tumor heterogeneity: causes and consequences. Biochim Biophys Acta. 2010;1805(1):105-17.

Mazor T, Pankov A, Johnson BE, Hong C, Hamilton EG, Bell RJA, et al. DNA Methylation and Somatic Mutations Converge on the Cell Cycle and Define Similar Evolutionary Histories in Brain Tumors. Cancer Cell. 2015;28: 307-17.

Mazor T, Pankov A, Song JS, Costello JF. Intratumoral Heterogeneity of the Epigenome. Cancer Cell. 2016;29:440-51.

McGranahan N, Swanton C. Clonal Heterogeneity and Tumor Evolution: Past, Present, and the Future. Cell. 2017;168:613-28.

Meacham CE, Morrison SJ. Tumour heterogeneity and cancer cell plasticity. Nature. 2013; 501:328-37.

Meyer N, Penn LZ. Reflecting on 25 years with MYC. Nat Rev Cancer. 2008;8:976-90.

Morel F, Le Bris MJ, Herry A, Le Calvez G, Marion V, Abgrall JF, et al. Double minutes containing amplified bcr-abl fusion gene in a case of chronic myeloid leukemia treated by imatinib. Eur J Haematol. 2003;70:235-9.

Murtaza M, Dawson SJ, Tsui DWY, Gale D, Forshew T, Piskorz AM, et al. Non-invasive analysis of acquired resistance to cancer therapy by sequencing of plasma DNA. Nature. 2013; 497:108-12.

Murtaza M, Dawson SJ, Pogrebniak K, Rueda OM, Provenzano E, Grant J, et al. Multifocal clonal evolution characterized using circulating tumour DNA in a case of metastatic breast cancer. Nat Commun. 2015;6:8760-6.

Nielsen JL, Walsh JT, Degen DR, Drabek SM, McGill JR, Von Hoff DD. Evidence of gene amplification in the form of double minute chromosomes is frequently observed in lung cancer. Cancer Genet Cytogenet. 1993;65:120-4.
Nik-Zainal S, Alexandrov LB, Wedge DC, Van Loo P, Greenman CD, Raine K, et al. Mutational processes molding the genomes of 21 breast cancers. Cell. 2012;149:979-93.

Notta F, Mullighan CG, Wang JCY, Poeppl A, Doulatov S, Phillips LA, et al. Evolution of human BCR-ABL1 lymphoblastic leukaemiainitiating cells. Nature. 2011;469(7330):3627.

Nowell PC. The clonal evolution of tumor cell populations. Science. 1976;194(4260):23-8.

Ott G, Rosenwald A, Campo E. Understanding MYC-driven aggressive B-cell lymphomas: Pathogenesis and classification. Hematology Am Soc Hematol Educ Program. 2013;2013: $575-83$

Ozer ME, Sarica PO, Arga KY. New Machine Learning Applications to Accelerate Personalized Medicine in Breast Cancer: Rise of the Support Vector Machines. OMICS. 2020; 24(5):241-6.

Pfeifer GP, You YH, Besaratinia A. Mutations induced by ultraviolet light. Mutat Res. 2005; 571:19-31.

Pietarinen PO, Eide CA, Ayuda-Duran P, Potdar $S$, Kuusanmäki H, Andersson EI, et al. Differentiation status of primary chronic myeloid leukemia cells affects sensitivity to BCRABL1 inhibitors. Oncotarget. 2017;8(14): 22606-15.

Plaks V, Kong N, Werb Z. The cancer stem cell niche: how essential is the niche in regulating stemness of tumor cells? Cell Stem Cell. 2015; 16(3):225-38

Pon JR, Marra MA. Driver and passenger mutations in cancer. Annu Rev Pathol. 2015;10: 25-50.

Proietti-De-Santis L, Balajee AS, Garcia CL, Pepe G, Worboys AM, Palitti F. Inhibition of p53, p21 and Bax by pifithrin-a does not affect UV induced apoptotic response in CS-B cells. DNA Repair (Amst). 2003;2(8):891-900.

Proietti-De-Santis L, Balzerano A, Prantera G. CSB: An emerging actionable target for cancer therapy. Trends Cancer. 2018;4(3):172-5.

Rausch T, Jones DTW, Zapatka M, Stütz AM, Zichner T, Weischenfeldt J, et al. Genome sequencing of pediatric medulloblastoma links catastrophic DNA rearrangements with TP53 mutations. Cell. 2012;148:59-71.

Rodríguez-Paredes M, Esteller M. Cancer epigenetics reaches mainstream oncology. Nat Med. 2011;17:330-9.

Schofield JD, Weightman B. New knowledge of connective tissue ageing. J Clin Pathol Suppl (R Coll Pathol). 1978;12:174-90.

Stephens PJ, Greenman CD, Fu B, Yang F, Bignell GR, Mudie LJ, et al. Massive genomic rearrangement acquired in a single catastrophic event during cancer development. Cell. 2011; 144:27-40.

Stratton MR, Campbell PJ, Futreal PA. The cancer genome. Nature. 2009;458:719-24.

Szendro IG, Franke J, De Visser JAGM, Krug J. Predictability of evolution depends nonmonotonically on population size. Proc Natl Acad Sci USA. 2013;110(2):571-6. 
Taylor BJM, Nik-Zainal S, Wu YL, Stebbings LA, Raine K, Campbell PJ, et al. DNA deaminases induce break-associated mutation showers with implication of APOBEC3B and 3A in breast cancer kataegis. Elife. 2013;2:e00534.

Thirlwell C, Will OCC, Domingo E, Graham TA, McDonald SAC, Oukrif D, et al. Clonality assessment and clonal ordering of individual neoplastic crypts shows polyclonality of colorectal adenomas. Gastroenterology. 2010; 138(4):1441-7.

Thomas L, Stamberg J, Gojo I, Ning Y, Rapoport AP. Double minute chromosomes in monoblastic (M5) and myeloblastic (M2) acute myeloid leukemia: Two case reports and a review of literature. Am J Hematol. 2004;77:55-61.

Tie J, Kinde I, Wang Y, Wong HL, Roebert J, Christie M, et al. Circulating tumor DNA as an early marker of therapeutic response in patients with metastatic colorectal cancer. Ann Oncol. 2015;26:1715-22.

Tubio JMC. Somatic structural variation and cancer. Brief Funct Genomics. 2015;14:339-51.

Umar A, Boland CR, Terdiman JP, Syngal S, de la Chapelle A, Rüschoff J, et al. Revised Bethesda
Guidelines for hereditary nonpolyposis colorectal cancer (Lynch syndrome) and microsatellite instability. J Natl Cancer Inst. 2004;96:261-8

Venkitaraman AR. Cancer susceptibility and the functions of BRCA1 and BRCA2. Cell. 2002; 108:171-82.

Weller M, Stupp R, Reifenberger G, Brandes AA, Van Den Bent MJ, Wick W, et al. MGMT promoter methylation in malignant gliomas: Ready for personalized medicine? Nat Rev Neurol. 2010;6:39-51.

Wick W, Weller M, Van Den Bent M, Sanson M, Weiler M, Von Deimling A, et al. MGMT testing--the challenges for biomarker-based glioma treatment. Nat Rev Neurol. 2014;10:372-85.

Wright NA. Cell proliferation in carcinogenesis. In: Alison M, editor. Cancer Handbook. London: MacMillan Publishers; 2002.

Wu X, Northcott PA, Dubuc A, Dupuy AJ, Shih $\mathrm{DJH}$, Witt $\mathrm{H}$, et al. Clonal selection drives genetic divergence of metastatic medulloblastoma. Nature. 2012;482(7386):529-33.

Xu X, Hou Y, Yin X, Bao L, Tang a, Song L, et al. Single-cell exome sequencing reveals single- nucleotide mutation characteristics of a kidney tumor. Cell. 2012;148(5):886-95.

Yang L, Luquette LJ, Gehlenborg N, Xi R, Haseley PS, Hsieh C, et al. Diverse mechanisms of somatic structural variations in human cancer genomes. Cell. 2014;153:919-29.

Yates LR, Campbell PJ. Evolution of the cancer genome. Nat Rev Genet. 2012;13(11):795806 .

Yu M, Stott S, Toner M, Maheswaran S, Haber DA. Circulating tumor cells: Approaches to isolation and characterization. J Cell Biol. 2011;192:373-82.

Zhang F, Khajavi M, Connolly AM, Towne CF, Batish SD, Lupski JR. The DNA replication FoSTeS/MMBIR mechanism can generate genomic, genic and exonic complex rearrangements in humans. Nat Genet. 2009;41:84953.

Zhang H, Wang Y, Yang H, Huang Z, Wang X, Feng W. TCF7 knockdown inhibits the imatinib resistance of chronic myeloid leukemia K562/G01 cells by neutralizing the Wnt/ $\beta$ catenin/TCF7/ABC transporter signaling axis. Oncol Rep. 2020;45(2):557-68. 